\title{
We Asked the Experts: The Role of Rural Hospitals in Achieving Equitable Surgical Access in Low-Resourced Settings
}

\author{
Kathryn Chu ${ }^{1,2}$ (1) $\cdot$ Rebecca Maine $^{3} \cdot$ Riaan Duvenage $^{4,5}$
}

Accepted: 25 July 2021/Published online: 2 August 2021

(C) Société Internationale de Chirurgie 2021

\begin{abstract}
Strengthening and defining the role of rural hospitals within a surgical ecosystem is essential to improving quality and timely surgical access for rural people in low and middle-income countries (LMICs). Regional hospitals are the cornerstone of LMIC rural surgical care but have insufficient human resources and infrastructure that limit the surgical care they can provide. District hospitals are most accessible for many rural patients but also have limited surgical capacity. In order to surgical access for rural people, both regional and district hospital surgical services must be strengthened. A strong relationship between regional and district hospitals through a hub and spoke model is needed. Regional hospital surgeons can support training and supervision for and referrals from district hospitals. Telemedicine can play a key role to leapfrog physical barriers and surgical specialist shortages. The changing demographics of surgical disease will continue to worsen the strain on tertiary hospitals where most subspecialists in LMICs work. The fewer rural patients who need to travel to urban referral and tertiary facilities for problems that can be managed at lower-level facilities, the better access to timely surgical care for all.
\end{abstract}

\section{Introduction}

Universal health coverage (UHC) by 2030 is key to attaining several Sustainable Development Goals and cannot be achieved without robust surgical health systems

Kathryn Chu

kchu@sun.ac.za

1 Centre for Global Surgery, Department of Global Health, Faculty of Medicine and Health Sciences, Stellenbosch University, Francie van Zijl Drive,

Tygerberg, Cape Town 7507, South Africa

2 Department of Surgery, University of Botswana, Gaborone, Botswana

3 Department of Surgery, University of Washington, Seattle, USA

4 Department of Global Health, Ukwanda Centre for Rural Health, Stellenbosch University, Cape Town, South Africa

5 Department of Surgery, Worcester Hospital, Worcester, South Africa to provide safe, affordable, timely surgical care for all people. These goals are particularly challenging for rural communities in low- and middle-income countries (LMICs) where vulnerable populations such as the poor and marginalized live. Limited access to quality surgical care contributes to perioperative mortality in LMICs that exceeds the global average [1]. The lack of access to quality surgical care is most pronounced in rural communities compared to urban areas. Strengthening and defining the role of rural hospitals within a surgical ecosystem is essential to improving quality and timely surgical access for rural people in LMICs.

Regional hospitals are the cornerstone of LMIC rural surgical care. Lack of pre-hospital transport and poor road conditions can delay reaching care at the regional hospital. Furthermore, it can make it nearly impossible for patients with time-critical conditions to reach the tertiary referral hospitals, located in urban centres sometimes hundreds of miles away. For example, general surgeons at regional hospitals must frequently care for head and thoracic trauma 
without the assistance of neurosurgeons or cardiothoracic surgeons, who only work at tertiary centres. LMIC regional hospitals should be strengthened with additional human and infrastructural resources so that they can (1) treat the majority of emergency surgical conditions without the need to transfer and (2) rapidly diagnose patients who need higher levels of care. The limited region hospital surgical workforce consists of 1 or 2 of each category: general surgeons, orthopaedic surgeons, obstetricians/gynaecologists and anaesthesiologists. Financial and professional incentives must be developed to both retain existing surgical staff and hire additional surgical specialists. Fully functioning radiology and laboratory services are needed in order for regional hospitals to operate independently and rapidly determine which patients must transfer. Currently, computer tomography scanners are rare at rural regional hospitals. Critical care is an essential component of surgical provision and this capacity is limited by shortages of physical units, necessary equipment and trained experts. The lack of these services was particularly felt during the COVID-19 pandemic when there was a paucity of ventilated beds in many rural LMIC facilities.

If surgical services in rural areas were only provided by regional hospitals, many people would lack two-hour access to emergency surgery, an indicator of timely surgical care. On the other hand, greater than $90 \%$ of Africans would have timely access to surgical care if it were available at district hospitals (DHs) according to modelling studies [2]. Surgical capacity at DHs is more limited than at regional hospitals, in part because DHs lack fully trained surgical and anaesthesia providers. Rural doctors in South Africa noted several barriers to scaling up surgical care in DHs including the lack of training, communication and support from regional hospitals and an ill-defined DH role in surgical provision [3]. Two key efforts could address these issues: 1) Ensure regional hospitals support DHs through a hub and spoke model and 2) Define DH surgical packages of care appropriate for the specific context and train DH non-specialist surgical and anaesthesia providers to do that work.

A strong relationship between regional and district hospitals is critical to increase surgical access. A hub and spoke model would ensure support for training, supervision and referrals between these hospital levels. DH surgical capacity should include the tools to make faster and more accurate diagnoses such as appropriate laboratory and radiology services. Telemedicine including mHealth could expedite DH referrals to regional hospitals for complex emergency conditions and cancer cases [4].

DH operative capacity can be expanded with appropriate training and oversight from regional hospital surgeons for key procedures through outreach, remote supervision and training. Regional hospital surgeons should consider regular outreach support to DH provider training, or provide in-reach training for $\mathrm{DH}$ doctors at their regional facilities. Several African countries have non-specialists surgical providers including family physicians, medical officers and non-physician clinicians who deliver certain essential operations at DHs. In many studies, these nonspecialists have equivalent outcomes in common procedures like caesarean sections [5]. An understanding of local surgical epidemiology and output is essential to develop a context-appropriate surgical package for DHs and not all DHs would have the same package.

The changing demographics of surgical disease will continue to worsen the strain on tertiary hospitals where most subspecialists in LMICs work. Surgical conditions from non-communicable diseases such as cancer and complications of diabetes mellitus and hypertension have increased as life expectancy improves. As the number of people with an increasing breadth of common surgical conditions grows, both regional hospitals and DHs must expand surgical services since tertiary hospitals cannot absorb all surgical patients from rural and urban populations. Increasing the number of people seeking surgical care at tertiary hospitals for more common conditions will further limit surgical care for both complex and common conditions across the region.

In conclusion, rural timely and safe surgical care needs a strong surgical ecosystem of doctors and surgeons at various levels to work together to provide care for the population. The political leadership to prioritize access to care is necessary to provide additional training and infrastructure as well as strong hospital communication and referral networks. Strengthening the access to surgical care at regional and district hospitals has the benefit of increasing access to care for underserved rural populations. The fewer rural patients who need to travel to urban referral and tertiary facilities for problems that can be managed at lower-level facilities, the better access to timely surgical care for all.

\section{References}

1. GlobalSurg Collaborative (2016) Mortality of emergency abdominal surgery in high-, middle- and low-income countries. Br J Surg 103:971-988

2. Juran S, Broer PN, Klug SJ, et al. (2018) Geospatial mapping of access to timely essential surgery in sub-Saharan Africa BMJ Glob Health 3: e000875

3. Chu KM, Naidu P, Hendriks HJ et al (2020) Surgical care at rural district hospitals in low- and middle-income countries: an essential component of universal health coverage. Rural Remote Health 20:5920

4. Booyse K, Swart O, Gouws J, et al. (2020) The effect of the introduction of an electronic booking system to appropriately prioritise gastroscopies at a regional hospital in South Africa 
5. Ashengo T, Skeels A, Hurwitz EJH et al (2017) Bridging the human resource gap in surgical and anesthesia care in low-resource countries: a review of the task sharing literature Hum Resour. Health 15:77
Publisher's Note Springer Nature remains neutral with regard to jurisdictional claims in published maps and institutional affiliations. 